\title{
Benefits of extended oral anticoagulation after first episode of pulmonary embolism
}

N one of the current clinical practice guidelines offers clear recommendations on the optimal duration of anticoagulation after pulmonary embolism. Previous studies have reported that extending anticoagulant therapy beyond 3-6 months in patients with unprovoked venous thromboembolism can reduce the risk of recurrence, but whether this benefit is maintained after discontinuation of treatment remains uncertain. Accordingly, Couturaud and colleagues conducted the multicentre, randomized, controlled PADIS-PE trial to assess whether a further 18 months of warfarin treatment in addition to the initial 6 months of treatment with anticoagulation therapy was beneficial or harmful compared with placebo in patients with a first episode of unprovoked pulmonary embolism.

The PADIS-PE trial investigators enrolled patients who had experienced a first episode of unprovoked pulmonary embolism (defined as symptomatic pulmonary embolism in the absence of major risk factors for venous thromboembolism within 3 months of diagnosis) and were treated with a vitamin $\mathrm{K}$ antagonist for 6 months. Patients with previously confirmed pulmonary embolism or bleeding during the initial 6-month anticoagulation treatment period were excluded from the study. Eligible patients were randomly assigned to receive either warfarin or placebo for 18 months, and all patients were followed up for a median of 24 months thereafter. The primary outcome measure was the composite of recurrent venous thromboembolism and major bleeding within the 18-month treatment period.

In total, 371 patients were included in the primary analysis; of these, 184 were randomly assigned to receive warfarin, and 187 were randomly allocated to receive placebo. During the 18-month treatment

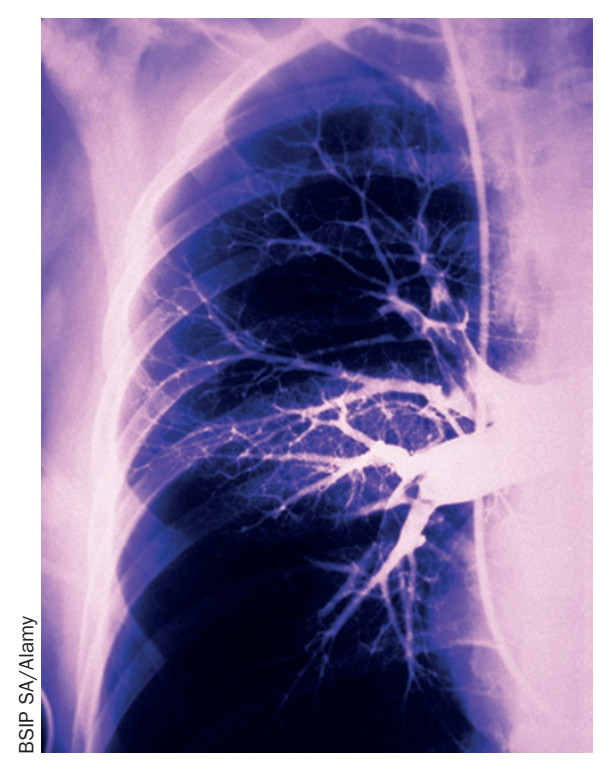

period, the primary outcome was observed in $3.3 \%$ of patients receiving warfarin, and $13.5 \%$ of patients receiving placebo. Warfarin treatment was associated with relative risk reduction of $78 \%$ (HR 0.22 , 95\% CI 0.09-0.55, $P=0.001)$. Recurrent venous thromboembolism occurred in three patients in the warfarin treatment group and 25 patients in the placebo group (HR 0.15, 95\% CI 0.05-0.43), whereas major bleeding was observed in four patients receiving warfarin and one patient receiving placebo (HR 3.96, 95\% CI 0.44-35.89). During the overall study period (18-month treatment period plus 24-month follow-up), the composite outcome was observed in $20.8 \%$ of patients treated with warfarin, and $24.0 \%$ of patients receiving placebo; this difference was not significant (HR 0.75, 95\% CI 0.471.18). These results indicate that, although additional warfarin therapy reduced the risk of recurrent venous thromboembolism during the treatment period, this benefit was not maintained after discontinuation of anticoagulant therapy.

Francis Couturaud, the lead investigator of the PADIS-PE trial, explains that before this study, the "optimal duration of anticoagulation after unprovoked venous thromboembolism was uncertain, and only two previous studies have suggested that extending anticoagulation for 1 year did not reduce the risk of recurrence". As in previous studies, the PADIS-PE investigators "observed a high absolute rate of recurrent venous thromboembolism in the placebo group during the 18-month treatment period". However, by the median 24-month post-treatment follow-up, the rate of recurrent venous thromboembolism in patients in the warfarin treatment group was approximately twice that in the placebo group, and was similar to the rates observed in the placebo group during the 18-month study treatment period. In addition, regardless of whether patients received 6 or 24 months of anticoagulant therapy, the clinical presentation of the majority of patients with recurrent venous thromboembolism was again in the form of symptomatic pulmonary embolism, with a death rate of $8 \%$.

Stavros Konstantinides (University Medical Center Mainz, Germany), who was not part of the study, believes that "the results of this trial support, stronger than ever before, the continuation of anticoagulation after pulmonary embolism for an indefinite period, showing that no predetermined time period of anticoagulation is safe to discontinue these drugs". He predicts that "optimized contemporary management of vitamin $\mathrm{K}$ antagonist treatment, or alternatively the good efficacy and safety profile of the new oral anticoagulants in this setting, will result in a growing number of patients receiving extended anticoagulation ... and will affect recommendations in future guidelines".

Karina Huynh

Original article Couturaud, F. et al. Six months vs extended oral anticoagulation after a first episode of pulmonary embolism: the PADIS-PE randomized clinical trial. JAMA 314, 31-40 (2015) 